\title{
Non-host class II ribonucleotide reductase in Thermus viruses: sequence adaptation and host interaction
}

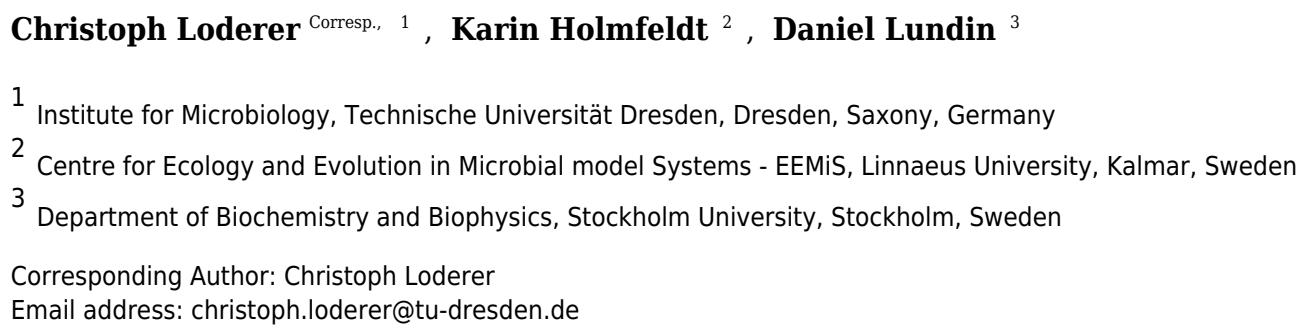

Ribonucleotide reductases (RNR) are essential enzymes for all known life forms. Their current taxonomic distribution suggests extensive horizontal gene transfer e.g. by processes involving viruses. To improve our understanding of the underlying processes, we characterized a monomeric class II RNR (NrdJm) enzyme from a Thermus virus, a subclass not present in any sequenced Thermus spp. genome. Phylogenetic analysis revealed a distant origin of the $n r d J m$ gene with the most closely related sequences found in mesophiles or moderate thermophiles from the Firmicutes phylum. GC-content, codon usage and the ratio of coding to non-coding substitutions (dN/dS) suggest extensive adaptation of the gene in the virus in terms of nucleotide composition and amino acid sequence. The NrdJm enzyme is a monomeric $B_{12}$-dependent RNR with nucleoside triphosphate specificity. It exhibits a temperature optimum at $60-70^{\circ} \mathrm{C}$, which is in the range of the growth optimum of Thermus spp. Experiments in combination with the Thermus thermophilus thioredoxin system show that the enzyme is able to retrieve electrons from the host NADPH pool via host thioredoxin and thioredoxin reductases. This is different from other characterized viral RNRs such as T4 phage RNR, where a viral thioredoxin is present. We hence show that the monomeric class II RNR, present in Thermus viruses, was likely transferred from an organism phylogenetically distant from the one they were isolated from, and adapted to the new host in genetic signature and amino acids sequence. 


\section{Non-host class II ribonucleotide reductase in Thermus viruses: sequence adaptation and host interaction}

Christoph Loderer ${ }^{*}$, Karin Holmfeldt ${ }^{2}$ Daniel Lundin ${ }^{3}$

${ }^{1}$ Institute for Microbiology, Technische Universität Dresden, Dresden, Saxony, Germany ORCID: https://orcid.org/0000-0002-3795-8250

${ }^{2}$ Centre for Ecology and Evolution in Microbial model Systems - EEMiS, Linnaeus University, Kalmar, Sweden

ORCID: https://orcid.org/0000-0002-6887-6661

${ }^{3}$ Department of Biochemistry and Biophysics, Stockholm University, Stockholm, Sweden ORCID: https://orcid.org/0000-0002-8779-6464

Corresponding Author:

Christoph Loderer 


\section{Non-host class II ribonucleotide reductase in Thermus}

3 viruses: sequence adaptation and host interaction

4

5

6

7

8

9

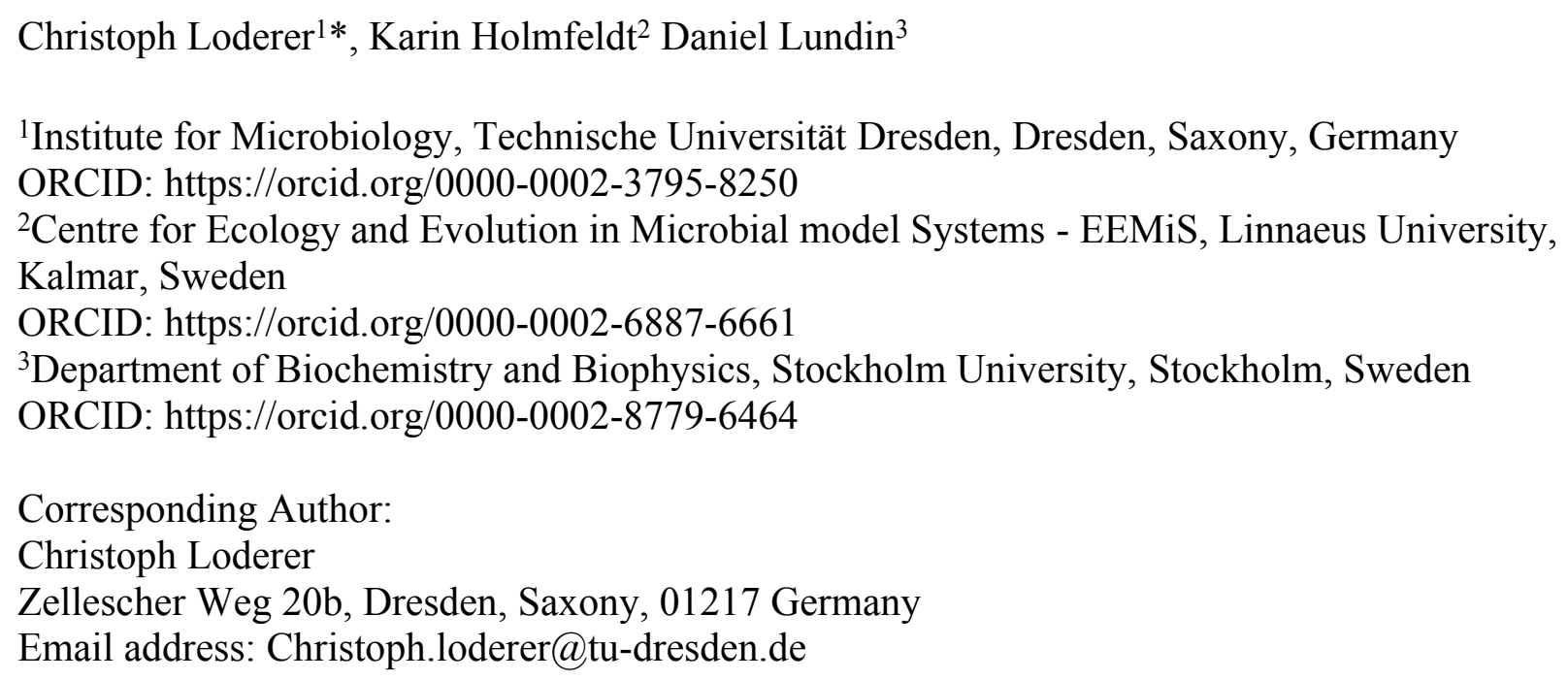

\section{ABSTRACT}

Ribonucleotide reductases (RNR) are essential enzymes for all known life forms. Their current taxonomic distribution suggests extensive horizontal gene transfer e.g. by processes involving viruses. To improve our understanding of the underlying processes, we characterized a monomeric class II RNR (NrdJm) enzyme from a Thermus virus, a subclass not present in any sequenced Thermus spp. genome. Phylogenetic analysis revealed a distant origin of the $\mathrm{nrdJm}$ gene with the most closely related sequences found in mesophiles or moderate thermophiles from the Firmicutes phylum. GC-content, codon usage and the ratio of coding to non-coding substitutions $(\mathrm{dN} / \mathrm{dS})$ suggest extensive adaptation of the gene in the virus in terms of nucleotide composition and amino acid sequence. The NrdJm enzyme is a monomeric $\mathrm{B}_{12}$-dependent $\mathrm{RNR}$ with nucleoside triphosphate specificity. It exhibits a temperature optimum at $60-70^{\circ} \mathrm{C}$, which is in the range of the growth optimum of Thermus spp. Experiments in combination with the Thermus thermophilus thioredoxin system show that the enzyme is able to retrieve electrons from the host NADPH pool via host thioredoxin and thioredoxin reductases. This is different from other characterized viral RNRs such as T4 phage RNR, where a viral thioredoxin is present. We hence show that the monomeric class II RNR, present in Thermus viruses, was likely transferred from an organism phylogenetically distant from the one they were isolated from, and adapted to the new host in genetic signature and amino acids sequence.

\section{INTRODUCTION}


42

43

44

45

46

47

48

49

50

51

52

53

54

55

56

57

58

59

60

61

62

63

64

65

66

67

68

69

70

71

72

73

74

75

76

77

78

79

80

81

Ribonucleotide reductases (RNRs) catalyze the reduction of ribonucleotides to the corresponding deoxyribonucleotides. Since this reaction is the only known biological de novo synthesis pathway for deoxyribonucleotides, it is essential to all known forms of life (Hofer et al. 2012; Lundin et al. 2015; Nordlund \& Reichard 2006). All known RNRs share a common reaction mechanism based on a catalytic thiyl radical and a common 10 stranded $\alpha / \beta$ fold. There are three different classes of RNRs that differ in features like radical generation mechanism, oligomeric state and terminal reductant (Hofer et al. 2012; Lundin et al. 2015; Nordlund \& Reichard 2006; Torrents 2014). Class I requires oxygen and is made up of a heterodimer consisting of an NrdA (catalytic subunit) homodimer and an NrdB (radical generating subunit) homodimer. Class II is a single component NrdJ protein that is vitamin $\mathrm{B}_{12}$-dependent. Most NrdJs are homodimeric, but the NrdJm subclass is monomeric. Class III is oxygen sensitive, both by the fact that oxygen cleaves the catalytic NrdD component in activated state and by the fact that the radical SAM family activase, NrdG, is oxygen sensitive (Lundin et al. 2015; Nordlund \& Reichard 2006). The organismal distribution of the different classes of RNRs does not follow the phylogeny of organisms but rather their biochemical requirements. Phylogenetic analysis suggests that multiple events of horizontal gene transfer (HGT), at phylogenetic distances varying from close relatives to inter domain transfers, are required to explain the distribution of RNRs (Lundin et al. 2010).

HGT can be mediated by viruses via transduction (Soucy et al. 2015). Indications of transduction-mediated interdomain HGT have been described for RNR in which a triplet of phylogenetically most closely related sequences were found in a halophilic bacterium (Salinibacter ruber), a halophilic archaeon (Natronomonas pharaonis) and a virus isolated from a saltern brine (Halophage AAJ-2005) (Lundin et al. 2010). However, to cover large phylogenetic distances, such as between phyla or domains, the process likely requires considerable evolution of host specificity in the virus, a process that is poorly known and mostly studied at relatively short phylogenetic distances (Koskella \& Meaden 2013). HGT by transduction is a stepwise process in which the transferred genes need to survive sufficiently long in the virus genome to come in contact with the receiving cellular organism during infection. In cases where genes transfer between distantly related cellular organisms, genes must remain in the virus during evolution of host specificity, suggesting that selection for a function provided by the transferred genes is likely to play a role. During their tenure in the viral genome, the transferred genes may also see selection to acquire a suitable GC content, codon usage and potential other genetic signatures. Ultimately, the transferred gene needs to either provide a selective advantage to its new host or by selfish processes ensure selection (Soucy et al. 2015). RNR is well suited to study these processes by being an essential enzyme - as it is required for DNA replication and repair - but is not an enzyme that is physically associated with a large multicomponent complex. Moreover, RNRs can be found in many double stranded DNA (dsDNA) viruses (Dwivedi et al. 2013; Sakowski et al. 2014) that can potentially act as vectors for transduction. 
82

83

84

85

86

87

88

89

90

91

92

93

94

95

96

97

98

99

100

101

102

103

104

105

106

107

108

109

110

111

112

113

114

115

116

117

118

119

120

For a transferred RNR to survive the stepwise process from the moment of transfer to potential selective advantage, the foremost functional requirement is the presence of a compatible reducing system in the receiving organism or in the infected host in the case of viruses. Three different systems to reduce the enzyme after catalysis have been found. In characterized class I and II, the reductant is thioredoxins or glutaredoxins (Booker \& Stubbe 1993; Holmgren \& Sengupta 2010), whereas in class III thioredoxin has been identified as the reductant in one enzyme (Wei et al. 2015) and formate in another (Mulliez et al. 1995). In the case of the Escherichia virus T4 (T4) NrdAB, the phage delivers a separate T4-thioredoxin that interacts with the native E. coli thioredoxin reductase (Berglund \& Holmgren 1975; Tseng et al. 1990). Other viruses, such as human Herpes simplex virus (HSV), do not encode viral thioredoxins and thus rely on their host's reduction system (Fawl \& Roizman 1994).

Adaptation of GC content and codon usage is an important aspect of genetic integration and genome evolution. This is true for RNRs not only in that the gene's coding sequence needs to adapt to overall GC content, as for all transferred genes, but also because RNR regulates the levels of the four dNTPs in cells (Hofer et al. 2012; Nordlund \& Reichard 2006). This is performed by allosteric substrate specificity regulation under the control of concentrations of individual dNTPs. In short, binding of a nucleotide - ATP, dATP, dCTP, dTTP and dGTP - to a specificity site, affects affinity for different nucleotides in the substrate binding pocket via the so called "loop 2" (Larsson et al. 2004). Imbalances in the dNTP pools are mutagenic (Hofer et al. 2012; Mathews 2014) and can hence potentially lead to the demise of a genetic lineage in extreme cases.

Most viral RNRs are similar to one found in their host organisms. For instance, T4-NrdA amino acid sequence is $54 \%$ identical to Escherichia coli NrdA. Thus, many viruses may have retrieved their RNRs from their corresponding host genomes. But there are exceptions. Screening the database RNRdb (http://rnrdb.pfitmap.org) for RNRs in viruses with no corresponding gene in the presumed host, we found three Thermus viruses (TV) with $n r d J m$ genes, encoding monomeric class II RNRs, not present in their hosts. By analyzing the phylogenetic history and sequence signatures of these genes, we traced back the evolutionary origin and investigated the process of genetic adaptation. In a biochemical characterization we investigated the functionality of the enzyme with respect to native conditions as well as its interaction with the host thioredoxin system.

\section{MATERIALS \& METHODS}

\section{Bioinformatics}


121

122

123

124

125

126

127

128

129

130

131

132

133

134

135

136

137

138

139

140

141

142

143

144

145

146

147

148

149

150

151

152

153

154

155

156

157

158

159

160

All NrdJm amino acid sequences from NCBI's RefSeq database were downloaded from RNRdb (http://rnrdb.pfitmap.org) and clustered with USEARCH (Edgar 2010) at 90\% sequence identity to reduce redundancy. Non full-length sequences and sequences of dubious quality were manually removed, before aligning all 364 sequences with ProbCons (Do et al. 2005).

Trustworthy alignment positions were selected with the BMGE algorithm (Criscuolo \& Gribaldo 2010) using the BLOSUM30 substitution matrix, ending up with 363 well-aligned positions forming 350 distinct alignment patterns. RAxML version 8.2.4 (Stamatakis 2014) was used to estimate a phylogeny, using the PROTGAMMAAUTO model, rapid bootstrapping with the autoMRE bootstopping followed by a full maximum likelihood tree search. The phylogeny is available at Figshare (https://doi.org/10.17045/sthlmuni.7117430.v1).

To search for sequences from metagenomes, we downloaded all TARA Ocean ORFs (https://doi.org/10.6084/m9.figshare.4902917,(Delmont et al. 2018)), all ORFs from the Human Microbiome Project (2017-01-09; (Human Microbiome Project 2012a; Human Microbiome Project 2012b)) the majority of archaeal and bacterial metagenome assembled genomes (MAGs) and single amplified genomes (SAGs) from IMG/MER (4910 MAGs, 2230 SAGs) plus 53 aquatic and soil metagenomes, in particular those with project names containing "virus", "phage", "therm" or "hot" (SI) (Markowitz et al. 2008). Together, we downloaded a total of $250,881,638$ ORFs. We used hmm profiles designed for each clan in the phylogeny to search the sequences. We found 181 sequences with a best match to the profile designed from the TV clan. These were aligned to the original alignment using Clustal Omega in profile mode (Sievers et al. 2011) and phylogenetically placed in the full phylogeny with RAxML (Stamatakis 2014) (https://doi.org/10.17045/sthlmuni.7642343.v1).

A $\log$ ratio test of significant overrepresentation of non-synonymous to silent (dN/dS) was estimated with codonml from PAML (Stamatakis 2014; Yang 1997; Yang 2007) by running the program with a fixed and free $\mathrm{dN} / \mathrm{dS}$ respectively, and the branch leading to the two Thermus virus sequences (marked with " $\# 1$ " in Figure 1B) designated as the "foreground" branch in the free $\mathrm{dN} / \mathrm{dS}$ run. This analysis was performed on the subtree in Figure 1B, using the same alignment as for the full protein sequence tree, reverse translated into the correct nucleotide sequence for each protein sequence. We could not find correct gene sequences for seven taxa (RefSeq accession numbers: WP_102410887, WP_033167051, WP_054955013, WP_065068364, WP_088370373, WP_087372021, WP_093315575), so they were left out of the analysis. (https://doi.org/10.17045/sthlmuni.7642463.v1)

The similarity of codon usage as euclidean distance between the codon frequencies was calculated as described elsewhere (Popa et al. 2017).

The model of the TV P74-26 nrdJm (TVNrdJm) was constructed with SwissModel (Waterhouse 
161

162

163

164

165

166

167

168

169

170

171

172

173

174

175

176

177

178

179

180

181

182

183

184

185

186

187

188

189

190

191

192

193

194

195

196

197

198

199

200

et al. 2018) using the Lactobacillus leichmannii monomeric NrdJ as template (PDB: 1L1L)

(Sintchak et al. 2002) and default parameters.

\section{Recombinant gene expression and purification}

Recombinant expression of the TV P74-26 nrdJm (UniprotKB: A7XXH5) was performed with a synthetic gene in a pET28b(+) expression vector and E. coli BL21(DE3) expression strain. 800 $\mathrm{mL}$ of LB-medium, containing $30 \mu \mathrm{g} \mathrm{mL}^{-1}$ kanamycin, were inoculated from a preculture to a cell density of $\mathrm{OD}_{600}=0.1$ and incubated at $37^{\circ} \mathrm{C}$ and $130 \mathrm{RPM}$. At an optical density of 1.0 expression was induced by addition of IPTG to a final concentration of $0.1 \mathrm{mmol} \mathrm{L}^{-1}$. Expression was performed at $37^{\circ} \mathrm{C}$ and $130 \mathrm{RPM}$ for $4 \mathrm{~h}$. After cell lysis with lysozyme and sonication, the enzyme was purified by Ni-NTA affinity chromatography followed by size exclusion chromatography.

Thermus thermophilus thioredoxin gene trx1 (UniprotKB: Q72HU9) and the thioredoxin reductase $t r$ gene (UniprotKB: Q72HD8) were cloned from genomic DNA in a pET52b(+) expression vector with C-terminal His-Tag. Expression was performed in a $E$. coli BL21(DE3) expression strain. For all genes, $800 \mathrm{~mL}$ of LB-medium, containing $100 \mu \mathrm{g} \mathrm{mL}^{-1}$ ampicillin, were inoculated from a preculture to a cell density of $\mathrm{OD}_{600}=0.1$ and incubated at $37^{\circ} \mathrm{C}$ and 130 RPM. At an optical density of 1.0 expression was induced by addition of IPTG to a final concentration of $0.1 \mu \mathrm{mol} \mathrm{L}-1$. Expression was performed at $130 \mathrm{RPM}$ for $16 \mathrm{~h}$ at $37^{\circ} \mathrm{C}$ for $\operatorname{Trx} 1$ and at $20^{\circ} \mathrm{C}$ for TR and. After cell lysis with lysozyme and sonication, the enzyme was purified by Ni-NTA affinity chromatography followed by desalting. Details of the purification procedure are given in the supporting information. An SDS-PAGE showing the purified NrdJm, Trx1 and TR is attached in the supporting information (Figure S1).

Analytical size exclusion chromatography was performed on a Superdex ${ }^{\mathrm{TM}} 200$ 10/300 GL column. $500 \mu \mathrm{g}$ of TV P74-26 $\mathrm{nrdJm}$ were loaded on the column and eluted with SEC buffer (50 mmol L-1 tris- $\mathrm{HCl}, 300 \mathrm{mmol} \mathrm{L}^{-1} \mathrm{NaCl}, 1 \mathrm{mmol} \mathrm{L}^{-1} \mathrm{DTT}, \mathrm{pH}=8.0$ ) with a flow rate of $0.2 \mathrm{ml}$ $\min ^{-1}$.

\section{Ribonucleotide reductase activity assays}

If not stated differently, RNR activity was measured for the conversion of the substrate GTP to the corresponding product dGTP in the presence of a dTTP effector. The standard setup of the assay contains $50 \mathrm{mmol} \mathrm{L}^{-1}$ Tris $(\mathrm{pH}=8.0), 50 \mathrm{mmol} \mathrm{L}^{-1} \mathrm{MgCl}_{2}, 50 \mathrm{mmol} \mathrm{L}-1$ Dithiothreitol (DTT), 1 mmol L-1 dTTP, 1 mmol L ${ }^{-1}$ GTP, $10 \mu \mathrm{mol} \mathrm{L}^{-1}$ AdoCbl and 0.1-0.4 $\mu \mathrm{mol} \mathrm{L}^{-1} \mathrm{NrdJm}$. In assays containing redoxin DTT was omitted and $2.5 \mu \mathrm{mol} \mathrm{L}-1$ Trx $1,1 \mu \mathrm{mol} \mathrm{L}^{-1} \mathrm{TR}$ and $2 \mathrm{mmol}$ $\mathrm{L}^{-1}$ NADPH were added. The assays were performed in $50 \mu \mathrm{L}$ scale in three independent experiments for each condition. The assay was started by addition of the RNR and stopped after 
201

202

203

204

205

206

207

208

209

210

211

212

213

214

215

216

217

218

219

220

221

222

223

224

225

226

227

228

229

230

231

232

233

234

235

236

237

238

239

240

5 to $15 \mathrm{~min}$ by addition of $50 \mu \mathrm{L}$ Methanol and incubation at $50{ }^{\circ} \mathrm{C}$ for 5 minutes. After centrifugation and addition of $200 \mu \mathrm{L} \mathrm{dH}_{2} \mathrm{O}$, the samples were analyzed via UHPLC.

UHPLC analysis was performed on a Knauer Platinblue ${ }^{\circledR}$ UHPLC. $5 \mu$ L of sample were injected on a Luna Omega 1.6 um Polar C18 ${ }^{\circledR}$ column from Phenomenex. GTP/dGTP and UTP/dUTP were eluted with a linear $\mathrm{MeOH}$ gradient from $26-30 \%$ over $15 \mathrm{~min}$ in a $50 \mathrm{mmol} \mathrm{L}^{-1} \mathrm{KP}_{\mathrm{i}}$-buffer $(\mathrm{pH}=7.0)$ containing $0.25 \%(\mathrm{v} / \mathrm{v})$ tetrabutylammonium hydroxide. CTP/dCTP were eluted with a linear $\mathrm{MeOH}$ gradient over 15 min from 27-30\% the same buffer. ATP/dATP were eluted with a linear $\mathrm{MeOH}$ gradient over $15 \mathrm{~min}$ from 10-30\% in the buffer as described before. A complete table of retention times is given in the supporting information (Table S17).

\section{RESULTS}

\section{Gene identification and phylogenetic analysis}

Screening the RNRdb (http://rnrdb.pfitmap.org) for viral RNRs with no similar host RNRs, we found three NrdJm enzymes, i.e. monomeric class II RNR enzymes, from viruses infecting Thermus species. The viruses belong to the Siphoviridae family and were found in hot biotopes. Thermus virus P74-26 and P23-45 were isolated at Uzon caldera in Kamchatka (Minakhin et al. 2008; Yu et al. 2006). The similar Thermus virus g20c was isolated at Geyzer Valley, Kamchatka (Xu et al. 2017). The NrdJm protein from Thermus viruses P74-26 (TVNrdJm, txid466052) and P23-45 (txid466051) have 96\% identical amino acid sequences. Since the sequence from TV g20c contains a likely intein, we excluded this sequence from further study. The phylogenetically closest well characterized homologue, from which a crystal structure is available, is the NrdJm from Lactobacillus leichmannii (Booker \& Stubbe 1993; Sintchak et al. 2002). This is currently the only well studied NrdJm enzyme and is only distantly related to TVNrdJm (Figure 1A). Within the phylum Deinococcus-Thermus no NrdJm enzymes could be found in current databases. The most closely related enzyme found in Thermus spp. are dimeric NrdJ enzymes with no significant sequence similarity to the TV enzymes.

We investigated the evolutionary descent of the three viral nrdJm genes by phylogenetic analysis of their respective amino acid sequences by estimating a maximum likelihood phylogenetic tree of the full diversity of NrdJm sequences from NCBI's RefSeq database (Figure 1A). The two viral sequences grouped inside a well-supported ( $82 \%$ bootstrap support) clan consisting exclusively of Firmicutes sequences except for the viral sequences (Figure 1B). Searching for metagenomic sequences similar to those in the TVNrdJm clan, only resulted in sequences placed in the Firmicute half of the clan (https://doi.org/10.17045/sthlmuni.7642343.v1). The identity between the viral sequences and the Firmicutes sequences was 35-42\% at the amino acid level. At nucleotide sequence level the similarity was hardly detectable using the BLAST algorithm. 
241 The similarity to genes from Firmicutes was shared with five other genes from the virus, whereas

242 eleven genes were most similar to genes from Proteobacteria and eight from Deinococcus-

243 Thermus (Table S8).

244

245

246

Since the NrdJm protein sequences are most closely related to Firmicutes sequences, we wanted

247 to see if the same held true for the genetic signature of sequences. Therefore, we compared

248

249

250

251

252

253

254

255

256

257

258

259

260

261

262

263

264

265

266

267

268

269

270

271

272

273

274

275

276

277

278

279

280 codon usage and GC-content of the TV mrdJm genes to Thermus and Firmicutes species. In codon usage, measured in euclidean distance, TV nrdJm genes showed high similarities to the genomes of the Thermus species and lower similarities to Firmicutes (Figure 1C). Since the similarity between Firmicutes and the Thermus species on average is even lower, the TV $\mathrm{nrdJm}$ codon usage is intermediary between Thermus spp. and Firmicutes. The GC content of the viral genomes as well as the $n r d J m$ genes was $57-58 \%$ (Figure 1B), relatively close to the GC-content of the Thermus hosts at $67-69 \%$. For the Firmicutes harboring the most similar nrdJms, the GCcontent was, with the exception of three taxa around 60\%, between 27 and 54\% (Figure 1B). Thermus spp. are thermophilic organisms with optimal growth temperatures between 60 and $70^{\circ} \mathrm{C}$. Most of the relevant Firmicutes are mesophiles, but some are at least moderate thermophiles despite their low GC content. Herbinix hemicellulosilytica, for instance, has an optimal growth temperature of $55^{\circ} \mathrm{C}$ with a $\mathrm{GC}$ content of only $36 \%$ (Koeck et al. 2015) (Figure 1B).

To investigate if positive selection had played a role in the evolution of the Thermus viruses since their divergence from the common ancestor with the Firmicute sequences, we performed a test of the ratio of non-synonymous to silent nucleotide substitutions (dN/dS) on this branch (marked with "\#1" in Figure 1B) with codonml from PAML (Yang 1997; Yang 2007). We found a dN/dS ratio of 17.5 , significantly greater than 1 (p-value: 0.036 ) and hence suggesting positive selection for amino acid substitutions. A Bayes Empirical Bayes analysis discovered 40 amino acid positions with a $\geq 95 \%$ posterior probability of being under positive selection (Table S10). To gain some insight into the possible role of the 40 amino acids, we modeled the TVNrdJm on the Lactobacillus leichmannii monomeric NrdJm structure (Sintchak et al. 2002) (Model in the SI) and mapped the positions of the significant non conservative mutations on to the model. In total, 18 of these positions are located around the specificity site or on the dimer mimicking domain. Ten positions are located in the $\mathrm{B}_{12}$ binding domain and another five cluster in a patch on the surface of the protein (Figure 1D).

\section{Recombinant expression of nrdJm genes and oligomeric state}

The gene coding for TVNrdJm was synthesized commercially with codon optimization for recombinant expression in E. coli. High levels of soluble expression were obtained after only $4 \mathrm{~h}$ of expression at $37^{\circ} \mathrm{C}$. After purification with IMAC and SEC we obtained $40 \mathrm{mg}$ of pure enzyme per liter of culture. The enzyme eluted after $16.8 \mathrm{~mL}$ on the size exclusion column, 
281

282

283

284

285

286

287

288

289

290

291

292

293

294

295

296

297

298

299

300

301

302

303

304

305

306

307

308

309

310

311

312

313

314

315

316

317

318

319

320

corresponding to a molecular weight of $65 \mathrm{kDa}$. With a calculated molecular weight of $73 \mathrm{kDa}$, this corresponds to a monomeric state of the enzyme in solution (Figure S2).

\section{The viral $n r d J m$ gene encodes a functional $B_{12}$-dependent ribonucleotide reductase with high temperature optimum}

The purified TVNrdJm was able to reduce GTP to the corresponding deoxyribonucleotide but not GDP, defining it as a nucleoside triphosphate reductase. Equimolar concentrations of cofactor $\mathrm{B}_{12}$ were sufficient to support the reaction, while higher concentrations reduced the activity to $40 \%$ at $0.1 \mathrm{mmol} \mathrm{L}^{-1}$ (Figure $2 \mathrm{~A}$ ). Without the presence of cofactor $\mathrm{B}_{12}$, no reaction was observed.

As the enzyme was isolated from a virus with a thermophilic host, reaction temperatures were monitored over a temperature range from $25^{\circ} \mathrm{C}$ to $90^{\circ} \mathrm{C}$. Enzyme activity peaks between $60^{\circ} \mathrm{C}$ and $70^{\circ} \mathrm{C}$ with complete inactivation at higher temperatures (Figure $2 \mathrm{~B}$ ). The $\mathrm{pH}$ profile of the enzyme was investigated between $\mathrm{pH}$ values 5 and 9 . The $\mathrm{pH}$ optimum was found to be between 7 and 8 with about 50\% residual activity between 6.5 and 9 (Figure $2 \mathrm{C}$ ).

The allosteric regulation of TVNrdJm was investigated by a survey of the reduction of the four ribonucleotides ATP, CTP, GTP and UTP with the potential allosteric effectors dATP, dCTP, dGTP and dTTP. Without allosteric effector, no activity could be observed for any of the four substrates (Table 1). Significant activity was only present for the substrate/effector pairs ATP/dGTP, CTP/dATP, GTP/dTTP.

\section{Terminal reduction of $\mathbf{N r d J m}$ with artificial reducing agents and Thermus Thioredoxins}

Activity of TVNrdJm was tested with the artificial reductants DTT and TCEP. While DTT is able to reduce the active site cysteine pair directly, TCEP can only work via the C-terminal cysteine pair (Domkin \& Chabes 2014; Loderer et al. 2017). The NrdJm exhibited 33\% higher activity with TCEP compared to DTT at their respective maxima (Figure 3 A). For both reductants, the maximal activity was reached already at $2 \mathrm{mmol} \mathrm{L}^{-1}$. Higher concentrations lead to a reduction of the activity to about $50 \%$ for both reductants at $100 \mathrm{mmol} \mathrm{L}^{-1}$.

For the RNR catalyzed reaction in vivo, reducing equivalents are required in form of gluta- or thioredoxins. Since the TVs do not encode redoxins, we tested TVNrdJm with the thioredoxin/thioredoxin reductase system from the strain Thermus thermophilus. With the redoxin system, the viral NrdJm was active with a comparable reaction velocity to the artificial reductants (Figure 3B). The control reaction without thioredoxin also displayed some activity. This may have been caused by residual DTT in the reaction mixture coming from the enzyme stocks. The experiment also showed that the complete redox reaction chain from NADPH over 
321

322

323

324

325

326

327

328

329

330

331

332

333

334

335

336

337

338

339

340

341

342

343

344

345

346

347

348

349

350

351

352

353

354

355

356

357

358

359

360

thioredoxin reductase, thioredoxin and RNR to the final electron recipient GTP is functional in vitro.

\section{DISCUSSION}

We discovered three viruses isolated from Thermus spp. that contain monomeric NrdJm RNRs, not found in their hosts or in related organisms. In the Deinococcus-Thermus phylum, no NrdJm enzymes could be found, opening the question of the evolutionary origin of the viral enzymes and their role in a potentially ongoing horizontal transfer of NrdJm RNRs by transduction. Phylogenetic analysis of amino acid sequences of NrdJm RNRs showed that the closest related sequences are found in Firmicutes, most of which are mesophilic although we also discovered a small number of moderate thermophiles. Further, five additional viral genes, dispersed throughout the Thermus virus P74-26 genome (Table S8), potentially share the same history as they were more similar to genes from Firmicutes than anything else in the RefSeq database. This relatively large number of genes with highest similarity to Firmicutes or prophages integrated in Firmicutes genomes, suggest a distant evolutionary relation between the TV and Firmicutes. This could involve either a highly extended host range of the TV or one of its ancestors, or an evolutionary process where phages originally infecting Firmicutes (given the recruited NrdJm gene) have evolved a shift in host specificity. Given the limited host range seen for TV (Yu 2006) and that infectivity of viruses are typically limited even between strains of the same bacterial species and only a few phages have shown an ability to infect across genus (Sullivan et al. 2003) or class (Jensen et al. 1998) boundaries, it appears more likely that host shift played the most important role. Moreover, the large phylogenetic distance between the potential hosts, as well as a large number of TV genes most similar to genes in Proteobacteria and Actinobacteria (Table S8), suggests this was likely a lengthy process including intermediate hosts (Hendrix 2003; Hendrix et al. 1999). It should be taken into consideration that TV could potentially have been able to retrieve the NrdJm gene from another intermediate host, but given that no sequences of higher similarity to the TV NrdJm has been detected, this cannot be verified.

The convergence of codon usage and GC content of the TVNrdJm gen and the Thermus genomes, show host adaptation of the gene at DNA sequence level. But adaptation also took place at amino acid sequence level, shown by the high $\mathrm{dN} / \mathrm{dS}$ value between the branch leading to the Thermus virus compared with the rest of the TV/Firmicutes clan. The location of the 40 positions with significant positive selection hints at the traits, where selection took effect. The 18 amino acid substitutions with $\geq 95 \%$ posterior probability of being under positive selection, located in the proximity of the specificity site, are particularly suggestive as RNRs are responsible for the relative sizes of dNTP pools (Hofer et al. 2012; Mathews 2014; Nordlund \& Reichard 2006). This could be a sign of adaptation to a genome of higher GC-content - close to $60 \%$ in TV compared with $25-52 \%$ in the Firmicutes' genomes - not only by its coding 
361

362

363

364

365

366

367

368

369

370

371

372

373

374

375

376

377

378

379

380

381

382

383

384

385

386

387

388

389

390

391

392

393

394

395

396

397

398

399

400

sequence, but also by how substrate specificity of the enzyme is regulated. Like all RNRs studied so far - except for RNRs in some members of the Herpesviridae family (Averett et al. 1983) allosteric regulation of substrate specificity is intact in TVNrdJm and comparable to other described RNRs (Table 1) (Eliasson et al. 1999; Larsson et al. 2004; Loderer et al. 2017; Rozman Grinberg et al. 2018; Sintchak et al. 2002).

The TVNrdJm enzyme is a functional nucleoside triphosphate class II RNR, dependent on cofactor $\mathrm{B}_{12}$. The monomeric state in solution as well as the sequence similarity to L. leichmannii NrdJm suggest that TVNrdJm contains a dimer mimicking domain, as described for $L$. leichmannii (Sintchak et al. 2002). Moreover, the enzyme shows an intact allosteric regulation comparable to other described RNRs (Table 1). To our knowledge, this is only the second NrdJm to be characterized thoroughly. Because of the degree of divergence between what appears to be monomeric NrdJms (Fig. 1A), our characterization of a second member of this subclass confirms what was earlier only assumed as shared features, such as the monomeric oligomeric state and the triphosphate specificity (Lundin et al. 2015; Nordlund \& Reichard 2006).

The enzyme has a temperature optimum at $60-70^{\circ} \mathrm{C}$, which is in the range of the optimal growth temperatures of most Thermus species (Oshima \& Imahori 1974; Williams et al. 1996). Although our in vitro assay conditions do not fully reflect the in vivo conditions, we can still conclude that the required thermal stability is present. As potential native reducing equivalent, we found that Trx 1 from Thermus thermophilus can provide electrons for the nucleotide reduction. This is different from E. coli $\mathrm{T} 4$ phage, where a viral Thioredoxin is mediating the electron transfer (Berglund \& Holmgren 1975). Hence, the viral NrdJm enzyme is capable of directly tapping the host's NADPH pool for deoxyribonucleotide biosynthesis. Since Thermus species also possess a biochemical pathway for vitamin $\mathrm{B}_{12}$ biosynthesis, all biochemical requirements for the viral NrdJm to work in an infected Thermus cell are met (Jiang et al. 2013).

\section{CONCLUSIONS}

Thermus virus P74-26 obtained a class II RNR from a phylogenetically distant source and adapted it for functional expression in the viruses host strains. Signs of the adaptation processes at DNA level were found in codon usage and GC content, while a high $\mathrm{dN} / \mathrm{dS}$ value argues for positive selection at amino acid sequence level. Biochemical characterization revealed a functional, thermotolerant and monomeric RNR with the ability to retrieve electrons from its Thermus sp. host thioredoxin system. In contrast to the adaptation to the recent host, the details of its evolutionary descent remain enigmatic, limited by a lack of sequence information but also by our generally poor understanding of the evolution of viral host specificities.

Although not a completed transductive HGT yet, the mechanisms we describe in this article should be highly relevant for transduction events in general. The about 100-fold higher mutation 
401 rate of dsDNA viruses compared to bacteria could facilitate the adaptation process compared to 402 that in the microorganism itself (Lynch 2010; Sanjuan et al. 2010). Thus, the virus would not be 403 a mere vector for DNA transfer but the genetic equivalent of a pressure chamber, where genes 404 can become preadapted to a new environment. In our case, the described adaptation of an $\mathrm{nrdJm}$ 405 gene most similar to Firmicutes, to work for the virus in the Thermus host, could act as preadaptation for a possible, albeit not yet observed, transfer to the host itself. This mechanism can be imagined for any gene that is able to deliver a direct selective advantage for the virus and

\section{ACKNOWLEDGEMENTS}

We kindly acknowledge Eugen Schell and Gerda Claus for their lab work contributions and Britt-Marie Sjöberg for discussions. We also thank two anonymous reviewers whose suggestions improved the manuscript.

416

417

418

\section{REFERENCES}

419

420

421

422

423

424

425

426

427

428

429

430

431

432

433

434

435

436

437

438

439

440

441

442

443

444

Averett DR, Lubbers C, Elion GB, and Spector T. 1983. Ribonucleotide Reductase Induced by Herpes Simplex Type 1 Virus. The Journal of Biological Chemistry J258:9831-9838.

Berglund O, and Holmgren A. 1975. Thioredoxin reductase-mediated hydrogen transfer from Escherichia coli thioredoxin-(SH)2 to phage T4 thioredoxin-S2. The Journal of Biological Chemistry 250:2778-2782.

Booker S, and Stubbe J. 1993. Cloning, sequencing, and expression of the adenosylcobalamin-dependent ribonucleotide reductase from Lactobacillus leichmannii. Proceedings of the National Academy of Sciences of the United States of America 90:8352-8356. DOI: 10.1073/pnas.90.18.8352

Criscuolo A, and Gribaldo S. 2010. BMGE (Block Mapping and Gathering with Entropy): a new software for selection of phylogenetic informative regions from multiple sequence alignments. $B M C$ Evolutionary Biology 10:210. DOI: 10.1186/1471-2148-10-210

Delmont TO, Quince C, Shaiber A, Esen OC, Lee ST, Rappe MS, McLellan SL, Lucker S, and Eren AM. 2018. Nitrogen-fixing populations of Planctomycetes and Proteobacteria are abundant in surface ocean metagenomes. Nature microbiology

3:804-813. 10.1038/s41564-018-0176-9

Do CB, Mahabhashyam MSP, Brudno M, and Batzoglou S. 2005. ProbCons: Probabilistic consistencybased multiple sequence alignment. Genome Research 15:330-340. DOI: 10.1101/gr.2821705

Domkin V, and Chabes A. 2014. Phosphines are ribonucleotide reductase reductants that act via Cterminal cysteines similar to thioredoxins and glutaredoxins. Scientific Reports 4:5539. DOI: $10.1038 /$ srep05539

Dwivedi B, Xue B, Lundin D, Edwards RA, and Breitbart M. 2013. A bioinformatic analysis of ribonucleotide reductase genes in phage genomes and metagenomes. BMC Evolutionary Biology 13:1-17. DOI: $10.1186 / 1471-2148-13-33$

Edgar RC. 2010. Search and clustering orders of magnitude faster than BLAST. Bioinformatics 26:24602461. DOI: 10.1093/bioinformatics/btq461 
445

446

447

448

449

450

451

452

453

454

455

456

457

458

459

460

461

462

463

464

465

466

467

468

469

470

471

472

473

474

475

476

477

478

479

480

481

482

483

484

485

486

487

488

489

490

491

492

493

494

Eliasson R, Pontis E, Jordan A, and Reichard P. 1999. Allosteric control of three B12-dependent (class II) ribonucleotide reductases. Implications for the evolution of ribonucleotide reduction. The Journal of Biological Chemistry 274:7182-7189.

Fawl RL, and Roizman B. 1994. The molecular basis of herpes simplex virus pathogenicity. Seminars in Virology 5:261-271.

Hendrix RW. 2003. Bacteriophage genomics. Current Opinion in Microbiology 6:506-511. DOI: 10.1016/j.mib.2003.09.004

Hendrix RW, Smith MC, Burns RN, Ford ME, and Hatfull GF. 1999. Evolutionary relationships among diverse bacteriophages and prophages: all the world's a phage. Proceedings of the National Academy of Sciences of the United States of America 96:2192-2197. DOI: 10.1073/pnas.96.5.2192

Hofer A, Crona M, Logan DT, and Sjoberg BM. 2012. DNA building blocks: keeping control of manufacture. Critical Reviews in Biochemistry and Molecular Biology 47:50-63. DOI: $10.3109 / 10409238.2011 .630372$

Holmgren A, and Sengupta R. 2010. The use of thiols by ribonucleotide reductase. Free Radical Biology \& Medicine 49:1617-1628. DOI: 10.1016/j.freeradbiomed.2010.09.005

Human Microbiome Project C. 2012a. A framework for human microbiome research. Nature 486:215221. 10.1038/nature11209

Human Microbiome Project C. 2012b. Structure, function and diversity of the healthy human microbiome. Nature 486:207-214. 10.1038/nature11234

Jensen EC, Schrader HS, Rieland B, Thompson TL, Lee KW, Nickerson KW, and Kokjohn TA. 1998. Prevalence of Broad-Host-Range Lytic Bacteriophages of Sphaerotilus natans, Escherichia coli, and Pseudomonas aeruginosa. Applied and Environmental Microbiology 64:575-580.

Jiang L, Lin M, Li X, Cui H, Xu X, Li S, and Huang H. 2013. Genome Sequence of Thermus thermophilus ATCC 33923, a Thermostable Trehalose-Producing Strain. Genome Announcements 1. DOI: 10.1128/genomeA.00493-13

Koeck DE, Ludwig W, Wanner G, Zverlov VV, Liebl W, and Schwarz WH. 2015. Herbinix hemicellulosilytica gen. nov., sp. nov., a thermophilic cellulose-degrading bacterium isolated from a thermophilic biogas reactor. International Journal of Systematic and Evolutionary Microbiology 65:2365-2371. DOI: 10.1099/ijs.0.000264

Koskella B, and Meaden S. 2013. Understanding bacteriophage specificity in natural microbial communities. Viruses 5:806-823. DOI: 10.3390/v5030806

Larsson KM, Jordan A, Eliasson R, Reichard P, Logan DT, and Nordlund P. 2004. Structural mechanism of allosteric substrate specificity regulation in a ribonucleotide reductase. Nature structural \& molecular biology 11:1142-1149. DOI: 10.1038/nsmb838

Loderer C, Jonna VRC, Cona M, Rozman Grinberg I, Sahlin M, Hofer A, Lundin D, and Sjöberg BM. 2017. A unique cysteine-rich Zn-finger domain present in a majority of class II ribonucleotide reductases mediates catalytic turnover. The Journal of Biological Chemistry. DOI: 10.1074/jbc.M117.806331

Lundin D, Berggren G, Logan DT, and Sjoberg BM. 2015. The origin and evolution of ribonucleotide reduction. Life (Basel) 5:604-636. DOI: 10.3390/life5010604

Lundin D, Gribaldo S, Torrents E, Sjoberg BM, and Poole AM. 2010. Ribonucleotide reduction horizontal transfer of a required function spans all three domains. BMC Evolutionary Biology 10:383. DOI: $10.1186 / 1471-2148-10-383$

Lynch M. 2010. Evolution of the mutation rate. Trends in genetics 26:345-352. DOI: 10.1016/j.tig.2010.05.003

Markowitz VM, Ivanova NN, Szeto E, Palaniappan K, Chu K, Dalevi D, Chen IM, Grechkin Y, Dubchak I, Anderson I, Lykidis A, Mavromatis K, Hugenholtz P, and Kyrpides NC. 2008. IMG/M: a data management and analysis system for metagenomes. Nucleic acids research 36:D534-538. $10.1093 / \mathrm{nar} / \mathrm{gkm} 869$

Peer) reviewing PDF | (2018:10:31962:1:1:NEW 8 Feb 2019) 
495

496

497

498

499

500

501

502

503

504

505

506

507

508

509

510

511

512

513

514

515

516

517

518

519

520

521

522

523

524

525

526

527

528

529

530

531

532

533

534

535

536

537

538

539

540

541

542

543

544

545

Mathews CK. 2014. Deoxyribonucleotides as genetic and metabolic regulators. FASEB Journal 28:38323840. DOI: $10.1096 /$ fj.14-251249

Minakhin L, Goel M, Berdygulova Z, Ramanculov E, Florens L, Glazko G, Karamychev VN, Slesarev AI, Kozyavkin SA, Khromov I, Ackermann HW, Washburn M, Mushegian A, and Severinov K. 2008. Genome comparison and proteomic characterization of Thermus thermophilus bacteriophages P23-45 and P74-26: siphoviruses with triplex-forming sequences and the longest known tails. Journal of Molecular Biology 378:468-480. DOI: 10.1016/j.jmb.2008.02.018

Mulliez E, Ollagnier S, Fontecave M, Eliasson R, and Reichard P. 1995. Formate is the hydrogen donor for the anaerobic ribonucleotide reductase from Escherichia coli. Proceedings of the National Academy of Sciences of the United States of America 92:8759-8762.

Nordlund P, and Reichard P. 2006. Ribonucleotide reductases. Annual Review of Biochemistry 75:681706. DOI: 10.1146/annurev.biochem.75.103004.142443

Oshima T, and Imahori K. 1974. Description of Thermus thermophilus (Yoshida and Oshima) comb. nov., a Nonsporulating Thermophilic Bacterium from a Japanese Thermal Spa. International Journal of Systematic and Evolutionary Microbiology 24:102.112. DOI: 10.1099/00207713-24-1102

Popa O, Landan G, and Dagan T. 2017. Phylogenomic networks reveal limited phylogenetic range of lateral gene transfer by transduction. The ISME journal 11:543-554. DOI: 10.1038/ismej.2016.116

Rozman Grinberg I, Lundin D, Hasan M, Crona M, Jonna VR, Loderer C, Sahlin M, Markova N, Borovok I, Berggren G, Hofer A, Logan DT, and Sjöberg BM. 2018. Novel ATP-cone-driven allosteric regulation of ribonucleotide reductase via the radical-generating subunit. Elife:e31529. doi: 10.7554/eLife.31529.

Sakowski EG, Munsell EV, Hyatt M, Kress W, Williamson SJ, Nasko DJ, Polson SW, and Wommack KE. 2014. Ribonucleotide reductases reveal novel viral diversity and predict biological and ecological features of unknown marine viruses. Proceedings of the National Academy of Sciences of the United States of America 111:15786-15791. DOI: 10.1073/pnas.1401322111

Sanjuan R, Nebot MR, Chirico N, Mansky LM, and Belshaw R. 2010. Viral mutation rates. Journal of virology 84:9733-9748. DOI: 10.1128/JVI.00694-10

Sievers F, Wilm A, Dineen D, Gibson TJ, Karplus K, Li W, Lopez R, McWilliam H, Remmert M, Soding J, Thompson JD, and Higgins DG. 2011. Fast, scalable generation of high-quality protein multiple sequence alignments using Clustal Omega. Molecular systems biology 7:539. $10.1038 / \mathrm{msb} .2011 .75$

Sintchak MD, Arjara G, Kellogg BA, Stubbe J, and Drennan CL. 2002. The crystal structure of class II ribonucleotide reductase reveals how an allosterically regulated monomer mimics a dimer. Nature Structural Biology 9:293-300. DOI: 10.1038/nsb774

Soucy SM, Huang J, and Gogarten JP. 2015. Horizontal gene transfer: building the web of life. Nature Reviews Genetics 16:472-482. DOI: 10.1038/nrg3962

Stamatakis A. 2014. RAxML version 8: a tool for phylogenetic analysis and post-analysis of large phylogenies. Bioinformatics 30:1312-1313. DOI: 10.1093/bioinformatics/btu033

Sullivan MB, Waterbury JB, and Chisholm SW. 2003. Cyanophages infecting the oceanic cyanobacterium Prochlorococcus. Nature 424:1047-1051.

Torrents E. 2014. Ribonucleotide reductases: essential enzymes for bacterial life. Frontiers in cellular and infection microbiology 4:52. DOI: 10.3389/fcimb.2014.00052

Tseng MJ, He P, Hilfinger JM, and Greenberg GR. 1990. Bacteriophage T4 nrdA and nrdB Genes, Encoding Ribonucleotide Reductase, are Expressed both Speperately and Coordinately: Characterziation of NrdB Promotor. Journal of Bacteriology 172:6323-6332.

Waterhouse A, Bertoni M, Bienert S, Studer G, Tauriello G, Gumienny R, Heer FT, de Beer TAP, Rempfer C, Bordoli L, Lepore R, and Schwede T. 2018. SWISS-MODEL: homology modelling of protein structures and complexes. Nucleic Acids Research 46:W296-W303. DOI: 10.1093/nar/gky427 
546 Wei Y, Li B, Prakash D, Ferry JG, Elliott SJ, and Stubbe J. 2015. A Ferredoxin Disulfide Reductase

547

548

549

550

551

552

553

554

555

556

557

558

559

560

561

562

563

564 Delivers Electrons to the Methanosarcina barkeri Class III Ribonucleotide Reductase. Biochemistry 54:7019-7028. DOI: 10.1021/acs.biochem.5b01092

Williams RA, Smith KE, Welch SG, and Micallef J. 1996. Thermus oshimai sp. nov., isolated from hot springs in Portugal, Iceland, and the Azores, and comment on the concept of a limited geographical distribution of Thermus species. International Journal of Systematic Bacteriology 46:403-408. DOI: 10.1099/00207713-46-2-403

Xu RG, Jenkins HT, Chechik M, Blagova EV, Lopatina A, Klimuk E, Minakhin L, Severinov K, Greive SJ, and Antson AA. 2017. Viral genome packaging terminase cleaves DNA using the canonical RuvC-like two-metal catalysis mechanism. Nucleic Acids Research 45:3580-3590. DOI: 10.1093/nar/gkw1354

Yang Z. 1997. PAML: a program package for phylogenetic analysis by maximum likelihood. Bioinformatics:5.

Yang Z. 2007. PAML 4: phylogenetic analysis by maximum likelihood. Molecular biology and evolution 24:1586-1591. 10.1093/molbev/msm088

$\mathrm{Yu}$ MX, Slater MR, and Ackermann HW. 2006. Isolation and characterization of Thermus bacteriophages. Archives of virology 151:663-679. DOI: 10.1007/s00705-005-0667-x 


\section{Figure 1 (on next page)}

Phylogeny, sequence characteristics and growth optima.

(A) Unrooted maximum likelihood phylogeny of representative NrdJm amino acid sequences. The two virus sequences are indicated with an arrow. Phyla, with viruses assumed from their names to infect organisms from the same phylum, are indicated with coloured branches. The only solved structure from Lactobacillus leichmannii (PDB: 1L1L) is indicated with a small red circle. The root of the TV and Firmicutes clan is marked with a blue star. Full tree file available at Figshare ( https://doi.org/10.17045/sthlmuni.7117430.v1 ). (B) Detailed view of the clan containing the Thermus virus sequences, surrounded by a dashed line in A, with GC contents, optimum growth temperatures (when available; viruses set to typical host $70^{\circ} \mathrm{C}$ ), indications of temperature ranges (leaf node shape) and oxygen requirements (node colour). (C) NeighborNet depicting patterns of codon usage between Thermus thermophilus, Thermus viruses and selected Firmicutes measured in euclidean distances. A split containing Thermus spp. and TVs is encircled in red; the TVs are encircled in blue. (D) Positions of mutations with a posterior probability $\geq 95 \%$ to be positively selected, mapped on a homology model of TVNrdJm: red: specificity site, magenta: dimer mimicking domain, orange: proximity of the specificity site, green: B12 binding site. Blue: surface patch, cyan: others. 


\begin{tabular}{l} 
Actinobacteria \\
Bacteroidetes \\
Chlamydiae \\
Cyanobacteria \\
Eukaryota \\
Firmicutes \\
Planctomycetes \\
Proteobacteria \\
Spirochaetes \\
Thermus viruses \\
Verrucomicrobia \\
\hline
\end{tabular}

PeerJ
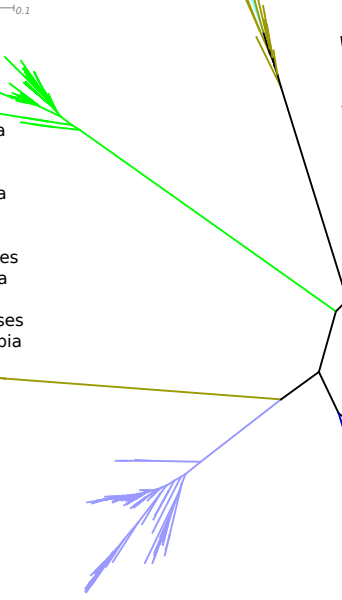

C

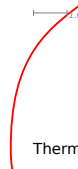

(-

Thermus thermophilus $\mathrm{HB} 2$

Thermus caldophilus

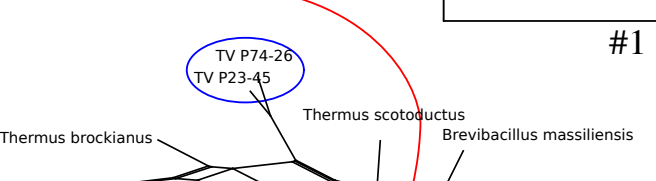

Thermus oshimai

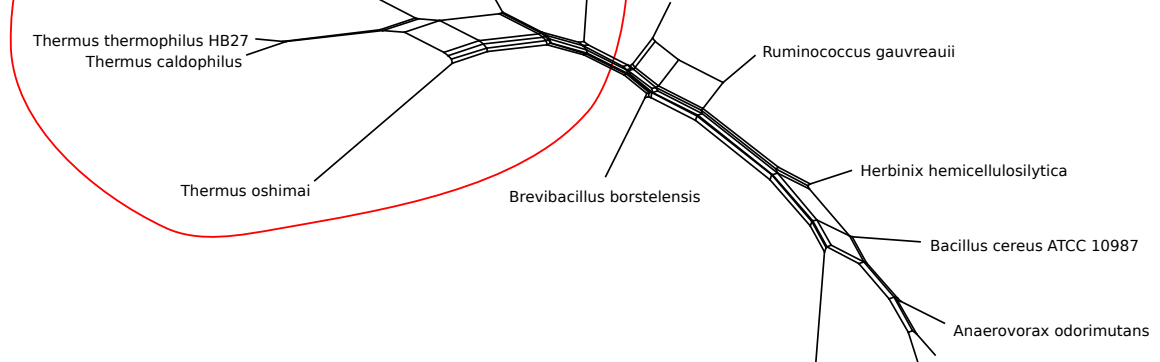

Thermus thermophilus Thermus oshimai Thermus caldophilus -
Thermus brockianus -

- Tindallia californiensis
epeptoclostridium acidaminophilum

Alkaliphilus transvaalensis
Alkaliphilus oremlandii

Akaliphilus metalliredigens

Anaeromicrobium sediminis
Alkaliphilus peptidifermentans

Inedibacterium massiliense Natronincola peptidivorans
Aneerovirgula multivorans lostridium formicaceticum . Acetoanaerobium noterae Clostridium sp. $\mathrm{C8}$
Clostridium fallax ethiosulfatibacter aminovorans
edimentibacter sp. B4 Acidaminobacter hydrogenoformans Anaerovorax odorimutans achnoclostridium sp. An196 Gemmiger sp. An120 Anaeromassilibacillus sp. An200
Clostridiales bacterium MarseilleP2846

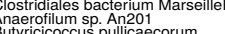
Butyricicoccus pullicaecorum Anaerovirgula multivorans Halanaerobium congolense Amphibacillus xylanus
Marinilactibacillus sp. $15 R$

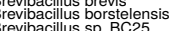
Brevibacillus massiliensis
B porosarcina koreensis Sporosarcina koreensis . Paenibacillus polymyxa Paenibacillus sp. FF9 Marininema halotolerans Brevibacillus laterosporus
Brevibacillus sp. SK 1010 Brevibacillus borstelensis
Bacillus sp. NSP2.1 Brevibacillus agri.1 Brevibacillus sp. WF146 Thssierellia bacreblum dichotomicum . Shimazuella kribbensis Clostridium felsineum
Clostridium sp. MarseilleP299 Anaerocolumna aminovaleric Bacillus cereus
Clostridium perfringens Clostridium uliginosum Clostridium chromiireducens Clostridium beijerinck Clostridium saccharobutylicum Herbinix hemicellllosilytica
Clostridium sp. KNHs205 Anaerocolumna xylanovoran Clostridioides difficile Thermus virus $P 742$

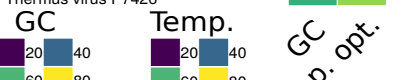

Microaerophilic Virus

D

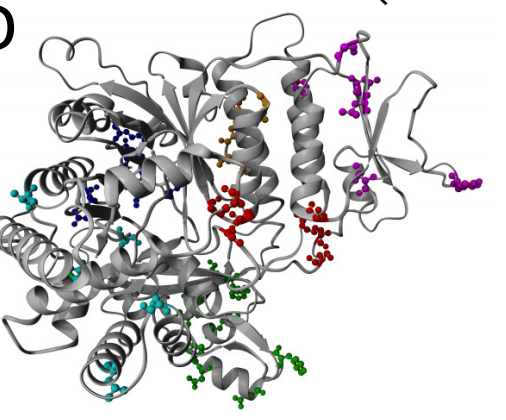


Figure 2

Biochemical characterization of TVNrdJm.

Effect of (A) B12-concentration (B) reaction temperature and (C) pH value on ribonucleotide reductase activity. Error bars represent the standard deviation of three independent experiments. 

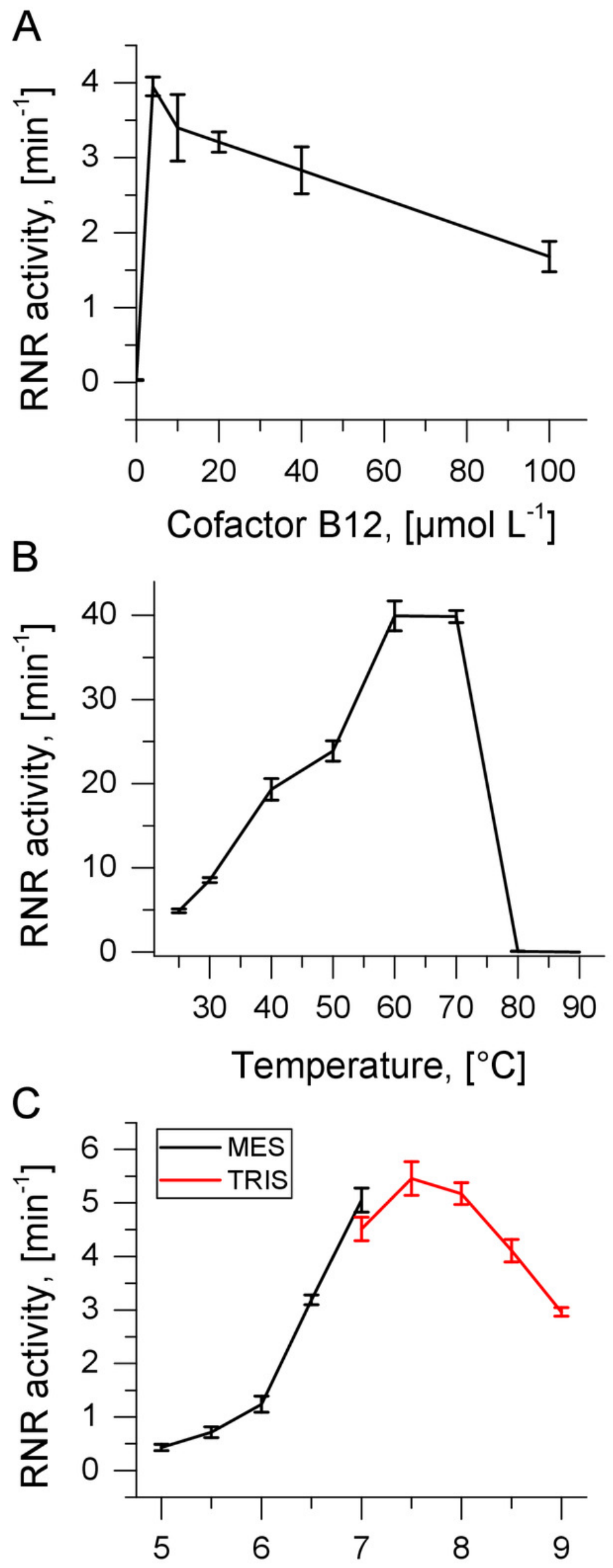
Figure 3

Artificial and potentially native reduction systems for NrdJm.

(A) Effect of the concentration of the artificial reductants DTT and TCEP on NrdJm activity. (B) NrdJm activity with thioredoxin Trx1 and thioredoxin reductase TR. Different control reactions were performed without thioredoxin and thioredoxin reductase. Error bars represent the standard deviation of three independent experiments.

A

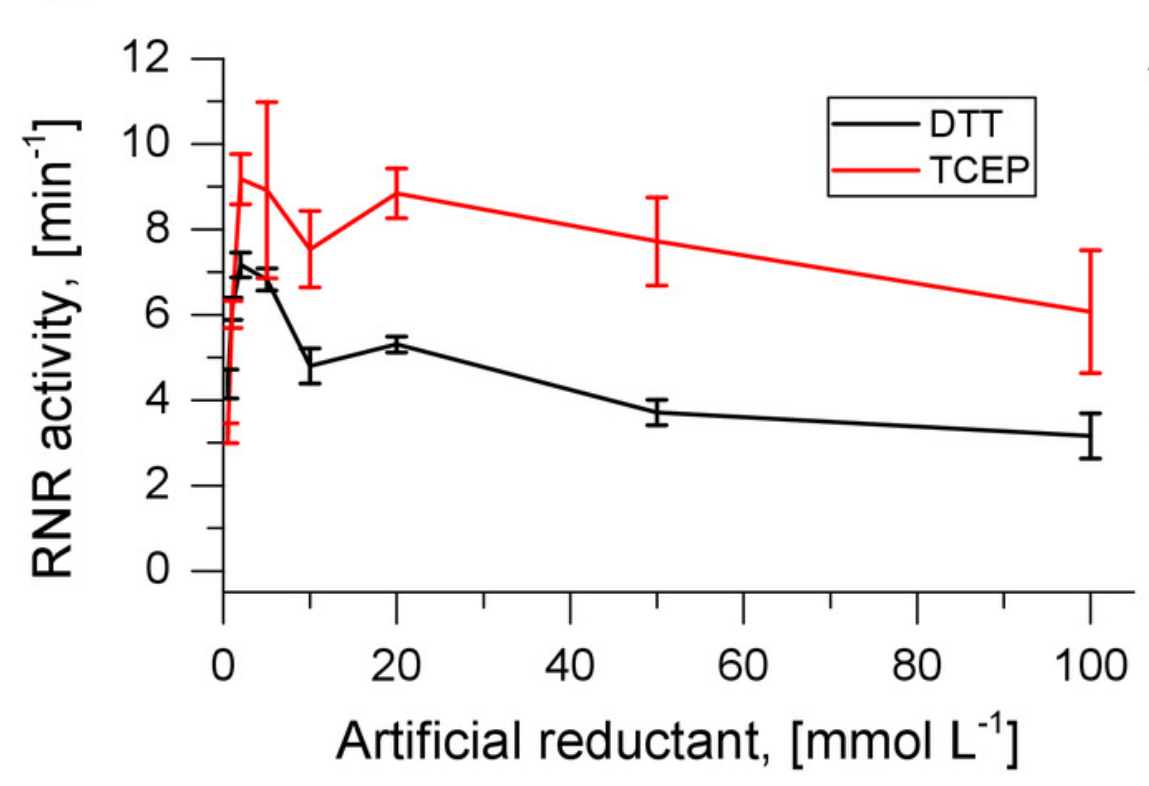

B

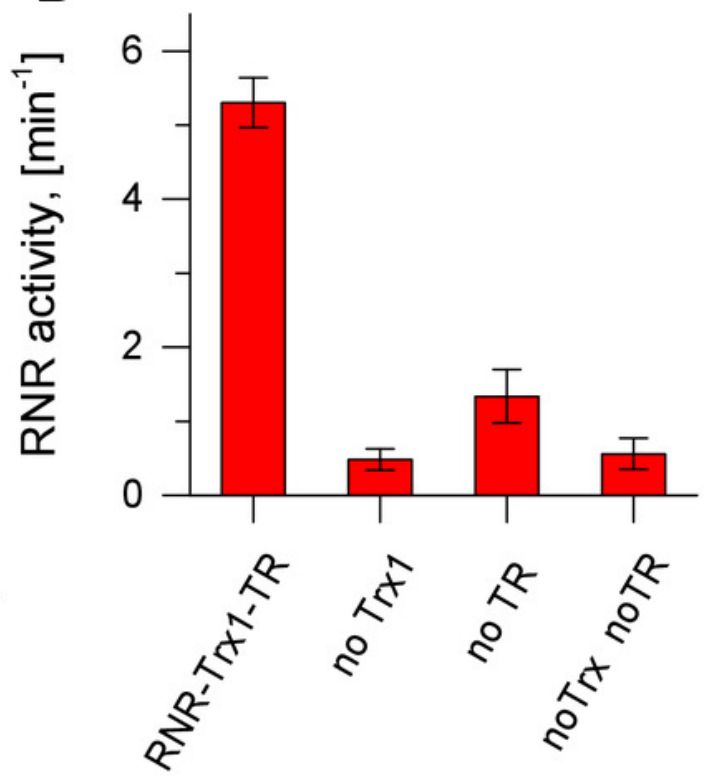




\section{Table $\mathbf{1}$ (on next page)}

Allosteric regulation of TVNrdJm.

RNR activity as kcat [min-1] for the four potential native substrates with dNTP effectors. n.d.: not determined, n.s.: no separation of effector and reaction product via HPLC. Errors indicate the standard deviation of three independent experiments. 
1

\begin{tabular}{|c|c|c|c|c|}
\hline & dATP & dCTP & dGTP & dTTP \\
\hline ATP & n. d. & $0.4 \pm 0.1$ & $2.2 \pm 0.3$ & $0.0 \pm 0.0$ \\
\hline CTP & $0.8 \pm 0.2$ & n. d. & $0.0 \pm 0.0$ & $0.0 \pm 0.0$ \\
\hline GTP & $0.1 \pm 0.0$ & $0.1 \pm 0.1$ & n. d. & $4.6 \pm 0.2$ \\
\hline UTP & $0.2 \pm 0.2$ & n.s. & n.s. & $0.0 \pm 0.0$ \\
\hline
\end{tabular}

2 